\title{
VARIATIONAL PRINCIPLES OF EQUILIBRIUM OF AN ELASTO-PLASTIC BODY*
}

\author{
BY \\ Y. YAMAMOTO \\ University of Tokyo
}

In this paper the author attempts to establish the general forms of the variational principles of equilibrium of an elasto-plastic body, and to make clear the relations among the principles presented previously [1]-[6].

$\$ 1$ Fundamental principle. Consider an elasto-plastic body ${ }^{1}$ which is stressed by the surface traction $F^{i}$ prescribed at each point on the portion $S_{F}$ of the surface $S$ of the body, the surface displacement $v_{i}$ prescribed at each point on the remaining portion $S_{v}$ of $S$, and the body force $K^{i}$ prescribed throughout the interior $V$ of the body. The stress and strain distributions in the body are assumed to be given by $\sigma^{i i}$ and $\epsilon_{i i}$ respectively. Then we may select some incremental stress-strain law $^{2}$ to hold at each point in the body. We assume that this law is such that any small possible change of the stress-strain state satisfies the condition

$$
\int_{0}^{\delta \epsilon} \delta \sigma^{i i} d \delta \epsilon_{i j} \geq 0^{3},
$$

which is a generalized form of the so-called uniqueness condition [7].

Now, suppose that the increment $\Delta F^{i}$ of the surface traction $F^{i}$ on $S_{F}$, the increment $\Delta v_{i}$ of the surface displacement $v_{i}$ on $S_{v}$ and the increment $\Delta K^{i}$ of the body force $K^{i}$ throughout $V$ are added gradually to the body. Then the resulting distribution of stress, strain and incremental displacement becomes $\sigma^{i i}+\Delta \sigma^{i j}, \epsilon_{i j}+\Delta \epsilon_{i j}$ and $\Delta u_{i}$ respectively. In the following lines we shall establish the variational principles which determine the resulting stress-strain state.

Since the resulting displacement and strain are usually small, we apply the infinitesimal deformation theory to our problem. An artificial displacement $\Delta u_{i}^{*}$, which is continuous and has piecewise continuous first and second derivatives, and which takes the prescribed value $\Delta v_{i}$ on $S_{v}$, is called an admissible displacement. Corresponding to it we may determine the strain $\Delta \epsilon_{i i}^{*}$ by the equation

$$
\Delta \epsilon_{i, j}^{*}=\frac{1}{2}\left(\Delta u_{i, j}^{*}+\Delta u_{i, i}^{*}\right)^{4} .
$$

Then the fundamental principle may be stated as follows: The following expression is non-negative for any artificial admissible displacement process $\Delta u_{i}^{*}(t)\left(t_{0} \leqq t \leqq t_{1}\right)$

\footnotetext{
*Received Oct. 29, 1951.

${ }^{1}$ We apply the Green's theorem in this body, so that its surface must have a suitable regularity.

${ }^{2}$ We need not assume one to one correspondence of the increments of stress and strain.

${ }^{3} \mathrm{As}$ usual the summation convention is used in this paper.

"Suffix ", $j$ " is the sign of differentiation by the ordinate $x^{i}$.
} 
such that $\Delta u_{i}^{*}\left(t_{0}\right)=\Delta u_{i}$, the actual displacement in the equilibrium state, and $\Delta u_{i}^{*}\left(t_{1}\right)=$ $\Delta u_{i}^{*}$, an admissible displacement;

$$
\begin{aligned}
\iiint d V \int_{\Delta \epsilon}^{\Delta \epsilon^{*}}\left(\sigma^{i j}+\Delta \sigma^{* i i}(t)\right) d \Delta \epsilon_{i j}^{*}(t)-\iiint & d V \rho\left(K^{i}+\Delta K^{i}\right)\left(\Delta u_{i}^{*}-\Delta u_{i}\right) \\
& -\iint_{S_{P}} d S\left(F^{i}+\Delta F^{i}\right)\left(\Delta u_{i}^{*}-\Delta u_{i}\right)^{5}
\end{aligned}
$$

where $\rho$ is the density of the material, the strain $\Delta \epsilon_{i j}^{*}(t)$ is derived by Eq. (2) from $\Delta u_{i}^{*}(t)$, and the stress $\Delta \sigma^{*^{i j}}(t)$ is determined by $\Delta \epsilon_{i j}^{*}(t)$ and the stress-strain law.

The expression (3) is equivalent to

$$
\begin{aligned}
\iiint d V\left(\sigma^{i j}+\Delta \sigma^{i j}\right) \delta \Delta \epsilon_{i j}^{*}-\iiint d V \rho\left(K^{i}+\Delta K^{i}\right) \delta \Delta u_{i}^{*} \\
-\iint_{S_{P}} d S\left(F^{i}+\Delta F^{i}\right) \delta \Delta u_{i}^{*}+\iiint d V \int_{0}^{\delta \Delta \epsilon^{*}} \delta \Delta \sigma^{*^{i j}}(t) d \delta \Delta \epsilon_{i j}^{*}(t),
\end{aligned}
$$

where $\delta \Delta u_{i}^{*}=\Delta u_{i}^{*}-\Delta u_{i}$, etc. By the principle of virtual work the first three terms in this expression vanish. Furthermore, the last term is always non-negative by Eq. (1). Thus, our proposition is proved.

\$2 Minimum principles. Unfortunately, the fundamental principle is not generally useful, because the value of the integral

$$
\int_{0}^{\Delta \epsilon^{*}}\left(\sigma^{i j}+\Delta \sigma^{*^{i j}}(t)\right) d \epsilon_{i j}^{*}(t)
$$

usually depends upon the stress-strain process. Hereafter we will assume that the value of this integral is determined only by the final values of $\Delta \sigma^{* i j}$ and $\Delta \epsilon_{i j}^{*}$ at each point in $v$, and that any stress-strain state is attainable from an arbitrary state. In such cases the following principle is clear.

Principle I: The expression

$$
\begin{aligned}
U+\Delta U^{*} \equiv \iiint d V \int_{0}^{\Delta \epsilon^{*}}\left(\sigma^{i i}\right. & \left.+\Delta \sigma^{* i j}(t)\right) d \Delta \epsilon_{i i}^{*}(t) \\
& -\iiint d V \rho\left(K^{i}+\Delta K^{i}\right) \Delta u_{i}^{*}-\iint_{S_{p}} d S\left(F^{i}+\Delta F^{i}\right) \Delta u_{i}^{*}
\end{aligned}
$$

takes its minimum value when the admissible displacement coincides with the actual one.

Certainly, the first variation becomes,

$$
\begin{aligned}
\delta\left(U+\Delta U^{*}\right)=\iiint d V\left(\sigma^{i j}+\Delta \sigma^{* i j}\right) \delta \Delta \epsilon_{i j}^{*} & -\iiint d V \rho\left(K^{i}+\Delta K^{i}\right) \delta \Delta u_{i}^{*} \\
& -\iint_{S_{P}} d S\left(F^{i}+\Delta F^{i}\right) \delta \Delta u_{i}^{*} .
\end{aligned}
$$

'Integration $\int_{\Delta \epsilon}^{\Delta \epsilon *} \ldots d \Delta \epsilon_{i j}^{*}(t)$ is taken along the process of the strain $\Delta \epsilon_{i j}^{*}(t)$. 
From this we obtain the well known equilibrium conditions of stress as follows:

$$
\left(\sigma^{i j}+\Delta \sigma^{* i j}\right),{ }_{j}+\rho\left(K^{i}+\Delta K^{i}\right)=0, \text { in } V,
$$

except on the discontinuity surface $S_{d}$ of stress in $V$,

$$
\left[\left(\sigma_{+}^{i j}+\Delta \sigma_{+}^{* i j}\right)-\left(\sigma_{-}^{i j}+\Delta \sigma_{-}^{* i j}\right)\right] m_{i}=0 \text {, on } S_{d},
$$

where $m_{j}$ is the unit normal vector on $S_{d}$, and $\sigma_{+}^{i j}$ and $\sigma_{-}^{i j}$ are the values of $\sigma^{i i}$ on the both sides of $S_{d}$, etc., and

$$
\left(\sigma^{i j}+\Delta \sigma^{*^{i j}}\right) n_{i}=F^{i}+\Delta F^{i}, \text { on } S_{F},
$$

where $n_{i}$ is the unit vector in the direction of the external normal of surface. The second variation becomes

$$
\delta^{2}\left(U+\Delta U^{*}\right)=\iiint d V \int_{0}^{\delta \Delta \epsilon^{*}} \delta \Delta \sigma^{*^{i i}}(t) d \delta \Delta \epsilon_{i j}^{*}(t)
$$

Since by Eq. (1) this value is non-negative, $U+\Delta U^{*}$ takes its minimum value when the admissible displacement coincides with the actual one.

By (2) we may easily obtain the equality

$$
\begin{aligned}
\iiint d V \sigma^{i j} \Delta \epsilon_{i j}^{*}-\iiint d V \rho K^{i} \Delta u_{i}^{*} & -\iint_{S_{F}} d S F^{i} \Delta u_{i}^{*} \\
& =\iint_{S_{v}} d S \sigma^{i j} n_{i} \Delta v_{i}=\text { const. } \equiv U
\end{aligned}
$$

Then the following principle may be obtained by Principle I and Eq. (9).

Principle I': The expression

$$
\Delta U^{*} \equiv \iiint d V \int_{0}^{\Delta \epsilon^{*}} \Delta \sigma^{*^{i j}}(t) d \Delta \epsilon_{i j}^{*}(t)-\iiint d V \rho \Delta K^{i} \Delta u_{i}^{*}-\iint_{S_{F}} d S \Delta F^{i} \Delta u_{i}
$$

takes its minimum value when the admissible state coincides with the actual one.

\$3 Maximum principles. Since the incremental strain is given by Eq. (2), the involute transformation of Principles $\mathrm{I}$ and $\mathrm{I}^{\prime}$ may be easily obtained by the general method [8] [9].

In the first place, Principle $I^{\prime}$ is equivalent to the following variational problem without any additional conditions,

$$
\begin{aligned}
\Delta U^{\prime} \equiv \iiint d V \int_{0}^{\Delta \epsilon^{* *}} \Delta \sigma^{* * i j}(t) d \Delta \epsilon_{i j}^{* *}(t) & \\
& -\iiint d V \rho \Delta K^{i} \Delta u_{i}^{* *}-\iint_{S_{P}} d S \Delta F^{i} \Delta u_{i}^{* *} \\
& -\iiint d V \lambda^{i j}\left[\Delta \epsilon_{i j}^{* *}-\frac{1}{2}\left(\Delta u_{i, j}^{* *}+\Delta u_{i, i}^{* *}\right)\right] \\
& -\iint_{S_{0}} d S \mu^{i}\left(\Delta u_{i}^{* *}-\Delta v_{i}\right)=\operatorname{Min} .
\end{aligned}
$$


where $\Delta u_{i}^{* *}$ is an artificial displacement which is continuous and piecewise differentiable, $\Delta \epsilon_{i j}^{* *}$ is an artificial strain which is independent of $\Delta u_{i}^{* *}$ and piecewise continuous, $\Delta \sigma^{* *^{i j}}$ is the stress corresponding to $\Delta \epsilon_{i j}^{* *}, \lambda^{i j}$ and $\mu^{i}$ are Lagrangian multipliers, and especially $\lambda^{i i}$ is piecewise continuous and differentiable. Then the natural conditions of Eq. (10) become

$$
\begin{aligned}
& \left.\begin{array}{rl}
\Delta \epsilon_{i j}^{* *} & =\frac{1}{2}\left(\Delta u_{i, i}^{* *}+\Delta u_{i, i}^{* *}\right), \text { in } V, \\
\Delta u_{i}^{* *} & =\Delta v_{i}, \text { on } S_{v},
\end{array}\right\} \\
& \Delta \sigma^{* * i j}=\lambda^{i j}, \text { in } V, \lambda^{i j} n_{j}=\mu^{i} \text {, on } S_{v} \text {, } \\
& \Delta F^{i}=\lambda^{i i} n_{i}, \text { on } S_{F}, \\
& \lambda_{, j}^{i i}+\rho \Delta K^{i}=0 \text {, in } V \text { except on the discontinuity surface } S_{d} \text { of } \lambda^{i i} \text {, and } \\
& \left(\lambda_{+}^{i j}-\lambda_{-}^{i j}\right) m_{i}=0 \text {, on } S_{d} \text {. }
\end{aligned}
$$

Since, if we add Eq. (11) to Eq. (10) as the additional conditions, Eq. (10) returns to Principle $I^{\prime}$, we substitute $\lambda^{i j}$ and $\mu^{i}$ into Eq. (10) from condition (12), i.e.,

$$
\begin{aligned}
\left.\Delta U^{\prime}\right|_{(12)}=-\iiint d V\left(\Delta \sigma^{* *^{i i}} \Delta \epsilon_{i j}^{* *}\right. & \left.-\int_{0}^{\Delta \epsilon^{* *}} \Delta \sigma^{* *^{i j}}(t) d \Delta \epsilon_{i j}^{* *}(t)\right) \\
& +\iint_{S_{v}} d S \Delta \sigma^{* *^{i j}} n_{j} v_{i} \\
=-\iiint d V \int_{0}^{\Delta \sigma^{* *}} \Delta \epsilon_{i j}^{* *}(t) d \Delta \sigma^{* *^{i j}}(t) & \\
& +\iint_{S_{v}} d S \Delta \sigma^{* * i j} n_{j} \Delta v_{i} \equiv \Delta U^{* *}, \quad \text { say. }
\end{aligned}
$$

Now, the value of the integral

$$
\int_{0}^{\Delta \sigma^{* *}} \Delta \epsilon_{i j}^{* *}(t) d \Delta \sigma^{* * i j}(t)=\Delta \sigma^{* * i j} \Delta \epsilon_{i j}^{* *}-\int_{0}^{\Delta \epsilon^{* *}} \Delta \sigma^{* *^{i j}}(t) d \Delta \epsilon_{i j}^{* *}(t)
$$

is also determined by the final values of $\Delta \sigma^{* *^{i i}}$ and $\Delta \epsilon_{i j}^{* *}$ only. Accordingly, we may regard $\Delta \sigma^{* *^{i j}}$ as the independent variable in the expression (13). When $\Delta \sigma^{* *^{i j}}$ is piecewise differentiable and satisfies the equilibrium conditions (6), (7), and (8), ${ }^{6}$ we call such a stress $\Delta \sigma^{* * i j}$ an admissible stress. Then, by the general theory of the involute transformation the following principle is valid.

Principle II': The expression

$$
\Delta U^{* *} \equiv-\iiint d V \int_{0}^{\Delta \sigma^{* *}} \Delta \epsilon_{i j}^{* *}(t) d \Delta \sigma^{* *^{i j}}(t)+\iint_{S_{v}} d S \Delta \sigma^{* *^{i j}} n_{j} \Delta v_{i}
$$

takes its maximum value when the admissible stress coincides with the actual stress.

${ }^{6}$ Because the conditions (12) correspond to the equilibrium condition. 
Certainly, we may confirm this principle as follows: Since, putting $\Delta \sigma^{* * i j}-\Delta \sigma^{i i}=$ $\delta \Delta \sigma^{* * i j}$, it is clear that, $\delta \Delta \sigma^{* * i j} n_{j}=\delta F^{i}=0$, on $S_{F}$,

$$
\delta \Delta \sigma_{, j}^{* *^{i j}}=-\delta\left(\rho K^{i}\right)=0 \text {, in } V,
$$

except on the discontinuity surface $S_{d}$ of $\delta \Delta \sigma^{* * i j}$, and

$$
\left(\delta \Delta \sigma_{+}^{* * i j}-\delta \Delta \sigma_{-}^{* *^{i j}}\right) m_{i}=0, \text { on } S_{d},
$$

we obtain the equality

$0=\iiint d V \Delta u_{i}^{* *} \delta \Delta \sigma_{, i}^{* *^{i j}}=-\iiint d V \Delta u_{i, j}^{* *} \delta \Delta \sigma^{* *^{i j}}+\iint_{S_{v}} d S \Delta u_{i}^{* *} n_{i} \delta \Delta \sigma^{* * i j}$

for any function $\Delta u_{i}^{* *}$ which is continuous and has piecewise continuous first derivatives. Then the first variation of $\Delta U^{* *}$ becomes,

$$
\begin{aligned}
\delta \Delta U^{* *} & =-\iiint d V \Delta \epsilon_{i i}^{* *} \delta \Delta \sigma^{* *^{i j}}+\iint_{S_{v}} d S \delta \Delta \sigma^{* * i j} n_{i} \Delta v_{i} \\
& =-\iiint d V\left(\Delta \epsilon_{i j}^{* *}-\Delta u_{i, j}^{* *}\right) \delta \Delta \sigma^{* * i j}+\iint_{S_{v}} d S \delta \Delta \sigma^{* *^{i j}} n_{j}\left(\Delta v_{i}-\Delta u_{i}^{* *}\right) .
\end{aligned}
$$

That is, as the natural condition of this principle, we obtain the fact that $\Delta \epsilon_{i j}^{* *}$ may be derived by Eq. (2) from suitable possible displacement $\Delta u_{i}^{* *}$. Furthermore the second variation of $\Delta U^{* *}$ becomes,

$$
\begin{aligned}
\delta^{2} \Delta U^{* *} & =-\iiint d V\left[\int_{0}^{\Delta \sigma^{* *}} \Delta \epsilon_{i j}^{* *}(t) d \Delta \sigma^{* * i j}(t)\right. \\
& \left.-\int_{0}^{\Delta \sigma} \Delta \epsilon_{i j}(t) d \Delta \sigma^{i i}(t)-\left(\Delta \sigma^{* * i j}-\Delta \sigma^{i j}\right) \Delta \epsilon_{i j}\right] \\
& =-\iiint d V \int_{0}^{\delta \Delta \sigma^{* *}} \delta \Delta \epsilon_{i j}^{* *}(t) d \delta \Delta \sigma^{* * i j}(t) .
\end{aligned}
$$

Since, we may attain to the stress-strain state $\left(\Delta \sigma^{i j}, \Delta \epsilon_{i j}\right)$ from the state $\left(\Delta \sigma^{* * i j}, \Delta \epsilon_{i j}^{* *}\right)$ by suitable process, we obtain the equalities

$$
\begin{aligned}
\int_{0}^{\delta \Delta \sigma^{* *}} \delta \Delta \epsilon_{i j}^{* *}(t) d \delta \Delta \sigma^{* * i i}(t) & =\delta \Delta \sigma^{* *^{i j}} \delta \Delta \epsilon_{i j}^{* *}-\int_{0}^{\delta \Delta \epsilon^{* *}} \delta \Delta \sigma^{* * i j}(t) d \delta \Delta \epsilon_{i j}^{* *}(t) \\
& =\int_{0}^{-\delta \Delta \epsilon^{* *}} \delta^{\prime} \Delta \sigma^{* *^{i j}}\left(t^{\prime}\right) d \delta^{\prime} \Delta \epsilon_{i j}^{* *}\left(t^{\prime}\right)
\end{aligned}
$$

where

$$
\delta^{\prime} \Delta \epsilon_{i j}^{* *}\left(t^{\prime}\right)=\Delta \epsilon_{i j}^{* *}\left(-t^{\prime}\right)-\Delta \epsilon_{i j}^{* *} .
$$

Accordingly, by condition (1) the value of $\delta^{2} \Delta U^{* *}$ is always non-positive. This shows that $\Delta U^{* *}$ generally takes its maximum value when the admissible stress coincides with the actual stress.

By Eq. (9), we may obtain the following principle. 
Principle II: The expression

$$
U+\Delta U^{* *}=-\iiint d V \int_{0}^{\Delta \sigma^{* *}} \Delta \epsilon_{i i}^{* *}(t) d \Delta \sigma^{* * i i}(t)+\iint_{S_{v}} d S\left(\sigma^{i j}+\Delta \sigma^{* * i i}\right) n_{i} \Delta v_{i}
$$

takes its maximum value when the admissible stress coincides with the actual.

From the general character of the involute transformation the following relations are obvious,

$$
\left.\begin{array}{l}
\text { Min. } \Delta U^{*}=\operatorname{Max} . \Delta U^{* *}, \quad \text { and } \\
\left(U+\Delta U^{*}\right)=\operatorname{Max} .\left(U+\Delta U^{* *}\right)=U+\operatorname{Min} . \Delta U^{*} .
\end{array}\right\}
$$

Certainly,

$$
\begin{aligned}
& \operatorname{Min} . \Delta U^{*}=\left.\Delta U^{*}\right|_{\Delta u^{*}=\Delta u_{i}} \\
& =\iiint d V \int_{0}^{\Delta \epsilon} \Delta \sigma^{i j}(t) d \Delta \epsilon_{i j}(t)-\iiint d V \rho \Delta K^{i} \Delta u_{i}-\iint_{S p} d S \Delta F^{i} \Delta u_{i} \\
& =\iiint d V \Delta \sigma^{i i} \Delta \epsilon_{i i}-\iiint d V \rho \Delta K^{i} \Delta u_{i}-\iint_{S_{F}} d S \Delta F^{i} \Delta u_{i} \\
& -\iiint d V\left(\Delta \sigma^{i i} \Delta \epsilon_{i j}-\int_{0}^{\Delta \epsilon} \Delta \sigma^{i j}(t) d \Delta \epsilon_{i j}(t)\right) \\
& =\iint_{S_{v}} d S \Delta \sigma^{i j} n_{i} \Delta v_{i}-\iiint d V \int_{0}^{\Delta \sigma} \Delta \epsilon_{i j}(t) d \Delta \sigma^{i i}(t) \\
& =\left.\Delta U^{* *}\right|_{\Delta \sigma^{* * i}=\Delta \sigma^{i i}}=\operatorname{Max} . \Delta U^{* *} \text {. }
\end{aligned}
$$

\$4 When are our principles valid? As already described our variational principles are valid when and only when the values of the integrals (5) and (14) are independent of the stress-strain process. Accordingly, when and only when the value of the integral

$$
\int_{0}^{\epsilon} \sigma^{i i} d \epsilon_{i j}
$$

is determined by the final values of stress and strain only, our principles are valid.

There are three reasons why the value of the integral (17) depends upon the stressstrain process: The first is the dependence of the stress-strain law upon the plastic history. Accordingly, we must assume that the stress-strain law is independent of the instantaneous plastic history. The second is the irreversibility of the stress-strain relation in case of the plastic deformation. That is, the stress-strain relations for loading and unloading states are individual ones. Then, our principles are valid for the cases where we may expect that the actual incremental process does not contain any unloading process at any yield surfaces except the initial one. Because in such cases we need not adopt the artificial admissible processes which contain such unloading processes. The third is the lack of the complete integrability of the integral (17) in stress space. We may easily obtain the general form of the stress-strain law which makes the integral 
(17) completely integrable if the unloading processes do not occur, and it becomes as follows:

$$
d \epsilon_{i j}=E_{i j k l} d \sigma^{k l}+F(f) \frac{\partial f}{\partial \sigma^{i j}}(d f+|d f|)\left(2 \sigma^{p q} \frac{\partial f}{\partial \sigma^{p q}}\right)^{-1},
$$

where $E_{i j k l}=E_{k l i j}$ is the elastic modulus, $f(\sigma)$ is the so-called loading function which is a function of $\sigma^{i j}$ only and the domain $f<$ const. $=c$ is the instantaneous elastic domain, and $F(f)$ is a positive function of $f(\sigma)$ only [10]. In this paper we assume such a stress-strain law. ${ }^{7}$

As above described, even if the stress strain law is given by Eq. (18), we can not assert the validity of our principles. But, if we can foresee that the stress-strain process, by which the resulting stress-strain state is attained, does not contain any unloading processes at any yield surfaces except the initial one $f(\sigma)=c_{0}$, where $c_{0}$ is determined by the plastic history just before the incremental deformation, we may form our principles for the admissible states which are attained by such processes. If we assume the following reversible stress-strain law which satisfies the condition (1), we may form such principles,

$$
\begin{aligned}
& d \Delta \epsilon_{i j}=E_{i j k l} d \Delta \sigma^{k l}, \quad \text { wherever } f<c_{0} \text {, or } f=c_{0} \text { but } d f \leq 0, \\
& =E_{i j k l} d \Delta \sigma^{k l}+F(f) \frac{\partial f}{\partial \sigma^{i j}} \frac{\partial f}{\partial \sigma^{k l}} d \Delta \sigma^{k l}\left[\left(\sigma^{p q}+\Delta \sigma^{p q}\right) \frac{\partial f}{\partial \sigma^{p q}}\right]^{-1}, \\
& \text { wherever } f>c_{0} \text {, or } f=c_{0} \text { but } d f>0 \text {, }
\end{aligned}
$$

where

$$
f=f\left(\sigma^{i i}+\Delta \sigma^{i j}\right) .
$$

The solution state of such principles satisfies the equilibrium and compatibility conditions, and by our assumption it is attainable by a suitable process whose stress-strain relation follows the Eq. (18), and which does not contain any unloading processes except those on the yield surface $f=c_{0}$. That is, in this case the actual resulting state is governed by these principles.

$\$ 5$ Principles for the stress and strain rates. Usually the mechanical quantities, such as strain, vary with finite time rates during a gradual stressing. In such cases they may be regarded to relate linearly with time during small time interval $\tau$, i.e.,

$$
\Delta \epsilon_{i j}^{*}(t)=\dot{\epsilon}^{*}(t), \quad \text { etc., } \quad(0 \leq t<\tau)
$$

except in the very small region whose volume vanishes with $\tau$. In the limiting case where $\tau$ tends to zero, we may regard that the process, by which the resulting state is attained, does not contain any unloading process except those on the initial yield surface. Then, as already described, our principles may be formed as principles for the rates. That is, they are easily obtained from Principles $\mathrm{I}^{\prime}$ and $\mathrm{II}^{\prime}$ as follows:

The expression

$$
\mathrm{I}_{a}^{\prime}: \quad \frac{1}{2} \ddot{U}^{*}=\iiint d V \frac{1}{2} \dot{\sigma}^{* i i} \dot{\epsilon}_{i j}^{*}-\iiint d V \rho \dot{K}^{i} \dot{u}_{i}^{*}-\iint_{S_{P}} d S \dot{F}^{i} \dot{u}_{i}^{*}
$$

'This is a generalized form of the Hodge-Prager stress-strain law [1]. 
takes its minimum value when the admissible displacement rate $\dot{u}_{i}^{*}$ coincides with the actual one.

The expression

$\mathrm{II}_{a}^{\prime}: \quad \frac{1}{2} \ddot{U}^{* *}=-\iiint d V \frac{1}{2} \dot{\sigma}^{* * i i} \dot{\epsilon}_{i j}^{* *}+\iint_{S_{v}} d S \dot{\sigma}^{* * i j} n_{j} \dot{v}_{i}$

takes its maximum value when the admissible stress rate $\dot{\sigma}_{i j}^{* *}$ coincides with the actual one.

The principle of Hodge and Prager is equivalent to our Principle $\mathrm{II}_{a}^{\prime}[1]$.

We may write down our principles for specific incremental stress-strain laws which belong to our general form (18). For example in the following lines we shall establish the principles for the material whose stress-strain relation follows the Prandtl-Reuss law, ${ }^{8}$

$$
\dot{\epsilon}_{i j}=\left(2 G_{0}\right)^{-1} \dot{s}^{i j}+\mu s^{i j}
$$

where

$$
\dot{s}^{i i}=\dot{\sigma}^{i j}-\frac{1}{3} \delta^{i j} \sigma^{k k}, \quad J_{2}=\frac{1}{2} s^{i i} s^{i j}
$$

and $\mu= \begin{cases}0 & \text { wherever } J_{2}<k^{2}, \text { or } J_{2}=k^{2} \text { but } \dot{J}_{2}<0, \\ \left(2 k^{2}\right)^{-1} s^{i j} \dot{\epsilon}_{i j}>0, & \text { wherever } J_{2}=k^{2} \text { and } \dot{J}_{2}=0 .\end{cases}$

In this case the following relations are easily obtained,

$$
\begin{aligned}
& \dot{\boldsymbol{\epsilon}}_{i i}=0, \quad \sigma^{i j} \dot{\boldsymbol{\epsilon}}_{i j}=s^{i i} \dot{\boldsymbol{\epsilon}}_{i j}=(2 G)^{-1} \dot{s}^{i j} s^{i j}+\mu s^{i j} s^{i j}, \\
& \dot{\sigma}^{i i} \dot{\boldsymbol{\epsilon}}_{i j}=2 G_{0}\left(\dot{\boldsymbol{\epsilon}}_{i j} \dot{\boldsymbol{\epsilon}}_{i j}-\mu s^{i j} \dot{\boldsymbol{\epsilon}}_{i j}\right)=\left(2 G_{0}\right)^{-1} \dot{s}^{i j} \dot{s}^{i j} \geq 0 .
\end{aligned}
$$

Then our principles become as follows:

The expression

$\mathrm{I}_{b}^{\prime}: \quad \frac{1}{2} \ddot{U}^{*}=\iiint d V G_{0}\left(\dot{\epsilon}_{i j}^{*} \dot{\epsilon}_{i j}^{*}-\mu^{*} s^{i j} \epsilon_{i j}^{*}\right)-\iiint d V \rho \dot{K}^{i} \dot{u}_{i}^{*}-\iint_{S F} d S \dot{F}^{i} \dot{u}_{i}^{*}$,

where

$$
\mu^{*}= \begin{cases}0, & \text { wherever } J_{2}<k^{2}, \text { or } J_{2}=k^{2} \text { but } s^{i j} \dot{\epsilon}_{i j}^{*} \leq 0, \\ \left(2 k^{2}\right)^{-1} S^{i i} \epsilon_{i i}^{*}, & \text { wherever } J_{2}=k^{2} \text { and } s^{i j} \dot{\epsilon}^{*}>0\end{cases}
$$

and the expression

$\mathrm{II}_{b}^{\prime}$ :

$$
\frac{1}{2} \ddot{U}^{* *}=-\iiint d V\left(4 G_{0}\right)^{-1} \dot{S}^{* * i j} \dot{s}^{* * i i}+\iint_{S_{v}} d S \dot{\sigma}^{* * i i} n_{j} \dot{v}_{i}
$$

take on minimum and maximum values respectively when the admissible rates coincide with the actual ones.

8The Prandtl-Reuss law and the Levy-Mises law belong to the Hodge-Prager's general type as the extreme cases [1].

${ }^{9}$ This condition corresponds to condition (1). 
The principles of Greenberg are equivalent to these principles [2] [3].

In the case where the elastic strain is very small compared with the plastic strain, we may neglect the elastic strain in the stress-strain law. In such cases our problem becomes somewhat complicated, because the stress state is not uniquely determined by a strain state. But the essential properties of our problem are unaltered by such circumstances. For example, we derive the principles for the material whose stress-strain relation follows the Levy-Mises law ${ }^{8}$ i.e.,

where

$$
d \epsilon_{i j}=\lambda s^{i j}
$$

$$
\lambda= \begin{cases}0, & \text { wherever } J_{2}<k^{2}, \text { or } J_{2}=k^{2} \text { but } d J_{2}<0 \\ \left(2 k^{2}\right)^{-1} s^{i j} d_{\epsilon i j}, & \text { wherever } J_{2}=k^{2} \text { and } d J_{2}=0 .\end{cases}
$$

In this case the increments of displacement and strain increase linearly with time, but the stress change is independent of time, i.e.,

$$
\Delta \epsilon_{i j}^{*}=\dot{\epsilon}_{i j}^{*}(t), \quad \Delta \sigma^{*^{i j}}=\text { const., etc., } \quad(0<t \leq \tau) .
$$

Then the following relations are obtained,

$$
\begin{gathered}
d \epsilon_{i j}=s^{i j}\left[d \epsilon_{p q} d \epsilon_{p q}\left(2 k^{2}\right)^{-1}\right]^{1 / 2}, \\
\int_{0}^{\Delta \epsilon^{*}}\left(\sigma^{i i}+\Delta \sigma^{* i j}(t)\right) d \Delta \epsilon_{i j}^{*}(t)=\left(\sigma^{i j}+\Delta \sigma^{* i j}\right) \Delta \epsilon_{i j}^{*}=\left(2 k^{2} \Delta \epsilon_{p q}^{*} \Delta \epsilon_{p q}^{*}\right)^{1 / 2} \\
\int_{0}^{\Delta \sigma^{* *}} \Delta \epsilon_{i j}^{* *}(t) d \Delta \sigma^{* * i j}(t)=0, \\
\int_{0}^{\delta \epsilon} \delta \sigma^{i j}(t) d \delta \epsilon_{i j}(t)=\delta \sigma^{i j} \delta \epsilon_{i j}=\delta s^{i j} s^{i j}\left[\delta \epsilon_{p q} \delta \epsilon_{p q}\left(2 k^{2}\right)^{-1}\right]^{1 / 2} \geq 0.9
\end{gathered}
$$

Then our principles are easily obtained by Principles I and II as follows:

The expression

$I_{c}$ :

$$
\dot{U}^{*}=\iiint d V\left(2 k^{2} \dot{\epsilon}_{p q} \dot{\epsilon}_{p q}\right)^{1 / 2}-\iiint d V \rho K^{i} \dot{u}_{i}^{*}-\iint_{S_{F}} d S F^{i} \dot{u}_{i}^{*}
$$

and the expression

$$
\mathrm{II}_{c}: \quad \dot{U}^{* *}=\iint_{S} d S\left(\sigma^{i j}+\Delta \sigma^{* * i j}\right) n_{j} \dot{v}_{i}
$$

take on minimum and maximum values respectively when the admissible displacement rate $\dot{u}_{i}^{*}$ and the admissible stress $\Delta \sigma^{* *^{i i}}$ coincide with the actual ones.

The principle of Markov and Hill and the principle of Sadowsky are equivalent to $I_{c}$ and $I I_{c}$ respectively [4].

$\$ 6$ Conclusion. The fundamental principle and Principle I are valid even in the case of finite deformations, but the other principles have an essential restriction in condition (2). ${ }^{10}$

${ }^{10}$ The Phillips' second principle seems to be dubious in some respects [ 6$]$. Involute transformation for finite deformation becomes very complicated. 
By the thermodynamical method we may prove the fundamental principle for stable equilibrium. Then the condition (1) seems to be essential in case of stable equilibrium. ${ }^{11}$ Moreover, it is notable that usually the condition (1) is equivalent to the uniqueness condition.

As described above our minimum and maximum principles are valid only in the restricted case. Accordingly, for the general cases we must apply the principle of virtual work directly, instead of the variational principle.

\section{Acknowledgement}

The author wishes to express his thanks to Professor W. Prager, Professor M. Yoshiki and Assistant Professor T. Kanazawa for constructive suggestions and criticism.

\section{BIBLIOGRAPHY}

[1] P. Hodge and W. Prager, A variational principle for plastic material with strain-hardening, J. Math Phys. 27, 4 (1949).

[2] H. J. Greenberg, Complementary minimum principles for an elastic-plastic material, Q. Appl. Math., 7, 1 (1949).

[3] W. Prager, Fundamental theorem of a new mathematical theory of plasticity, Duke Math. J., No. 1 (1942).

[4] R. Hill, A comparative study of some variational principles in the theory of plasticity, J. Appl. Mech., March (1950).

[5] A. H. Philippidis, The general proof of the principle maximum plastic resistance, J. Appl. Mech., 15, 3 (1948).

[6] A. Phillips, Variational principle in the theory of finite plastic deformation, Q. Appl. Math., 7, 1 (1949).

[7] W. Prager, Recent development in the mathematical theory of plasticity, J. Appl. Phys., 20, 3 (1949).

[8] R. Courant and D. Hilbert, Methoden der mathematischen Physik, 1 ter Bd., 2 te Auf., Kap. 4, 9, Julius Springer, Berlin (1931).

[9] M. Kotani, Mechanics of the continuous body, Lecture on Mathematics. Iwanami, Tokyo (in Japanese) (1939).

[10] Y. Yamamoto, A general theory on the plastic behavior of metals, to be published in Proc. First N.C.T.A.M. in Japan.

"IIf the condition (1) fails, new instability phenomena may occur. It seems to the author that it corresponds to fracture or yield. 